\title{
Guest Editorial: "Special issue on selected papers from NOSSDAV 2009”
}

\author{
Wei Tsang Ooi $\cdot$ Dongyan Xu
}

Published online: 15 May 2010

(C) Springer-Verlag 2010

We are pleased to introduce this special issue featuring selected papers from the 19th ACM Workshop on Network and Operating Systems Support for Digital Audio and Video (NOSSDAV 2009) held in Williamsburg, VA, USA. Based on the reviews of the NOSSDAV program committee, we invited authors of seven papers from the workshop to extend their papers for this special issue. We received five submissions, and, after a rigorous review process, three papers are accepted to appear in this special issue.

The first paper, entitled "Frequent Layer Switching for Perceived Quality Improvements of Coarse-Grained Scalable Video", addresses the issue of adapting the video data rate to the available bandwidth. Existing coding-based approach for fine-grain adaptation often lead to high overhead. In this paper, Pengpeng $\mathrm{Ni}$ and her co-authors investigate an alternative method to achieve fine-grain scalability with low coding overhead. They propose a technique called frequent layer switching, where a coarsegrain scalable video is switched between different layers frequently to adapt to available bandwidth. A key question is how such frequent layer switches affect the perceptual quality of the video. The authors, through extensive user studies, show that frequent layer switching does not significantly affect the perceptual quality under certain

\footnotetext{
W. T. Ooi $(\square)$

Department of Computer Science, National University of Singapore, Singapore, Singapore

e-mail: ooiwt@comp.nus.edu.sg

D. $\mathrm{Xu}$

Department of Computer Science and School of Electrical and Computer Engineering (courtesy), Purdue University, West Lafayette, IN, USA

e-mail: dxu@cs.purdue.edu
}

scenario and switching patterns, making it a viable approach to achieve fine-grain video rate adaptation to available bandwidth.

The second paper in this special issue considers the design of a large-scale VoD streaming system using peer-to-peer techniques. The paper, entitled, "InstantLeap: An Architecture for Fast Neighbor Discovery in Large-Scale P2P VoD Streaming," tackles the challenge of fast neighbor discovery whenever a VCR command is issued. In this paper, Xuanjia Qiu and co-authors, propose a method to organize peers to facilitate smooth playback when a peer seeks to a new playback position in the video. The key idea is for each peer to maintain, in addition to neighbors that are viewing the video around the same playback point, a set of shortcut neighbors whose playback points are further away. The authors show that these shortcut neighbors allow constanttime lookup of neighbors at the new playback position.

The last paper in this special issue, "Zebroid: Using IPTV Data to Support STB-Assisted VoD Content Delivery," considers scaling up VoD services in the context of IPTV. In this paper, the authors, Yih-Farn Robin Chen et al., propose that popular content be prefetched into selected set top boxes during idle hours. During peak hours, a set top box requesting a popular content can receive the content from participating neighborhood set top boxes, reducing the load on the VoD server. VoD access patterns, properties of set top boxes, and load on network components are considered in deciding how to partition the content into stripes and which stripe to store into which set top boxes. The paper shows that storing a small amount of data into each set top box can reduce the load on the VoD server significantly during peak hours.

The three selected papers cover many aspects of media streaming and distribution, from bandwidth adaptation, to reducing rebuffering time and improving scalability. We 
hope that the publication of these papers will stimulate broader interest and further research in the coming years.

We would like to thank all the contributing authors for preparing and submitting enhanced versions of their NOSSDAV papers to the special issue, and the reviewers for providing detailed reviews in a timely fashion. Finally, we would like to thank Reza Rejaie for his guidance throughout the editorial process of this special issue and Klara Nahrstedt, Chair of ACM SIGMM, for her continued support of the workshop and the special issue. 